\title{
MedienPädagogik
}

Zeitschrift für Theorie und Praxis der Medienbildung

Jahrbuch Medienpädagogik 2.

Zweitveröffentlichung aus: Jahrbuch Medienpädagogik 2. (2001) Opladen: Leske + Budrich. Herausgegeben von Ben Bachmair, Dieter Spanhel und Claudia de Witt.

\section{Neue Medien im erziehungswissenschaftlichen Studium}

Claudia de Witt

\section{Einleitung}

Heute wird mit dem Begriff „Neue Medien“ der vernetzte Computer verstanden, der Text-, Bild- und Tonmedien simuliert (vgl. Krämer 1998). Und neue Medien sorgen für eine veränderte Organisation des Lehrens und Lernens. Netzbasiertes Lehren und Lernen gehört derzeit zu den Top-Themen, die diskutiert werden. Die Implementierung netzbasierten Lehrens und Lernens in das erziehungswissenschaftliche Studium ist dagegen immer noch eher eine Seltenheit.

Die Diskussion über den Einsatz des Internets im Bildungswesen wird von neuen disziplinübergreifenden Modellen der Bildungsorganisation begleitet. Dazu gehören z.B. Zusammenschlüsse von Universitäten wie z.B. die Globewide Network Academy oder die Electronic Global University, die neue zentrale Vermittlungsstellen für OnlineBildung sind. Neue Bezeichnungen tauchen auf: Es wird gesprochen von Single-ModeUniversitäten und Dual-Mode-Universitäten. Single-Mode-Universitäten werden danach bestimmt, ob sie den Fernlehre-Ansatz fortsetzen und ihn erweitern. Dual-ModeUniversitäten erheben nicht den Anspruch, „nur“ als virtuelle Universitäten aufzutreten und mit Lernangeboten im Internet vertreten zu sein, sondern sie stellen Modelle mit einer Mischung aus traditionellen und hypermedialen Lehr- und Lernmethoden dar. Zu den Single-Mode-Universitäten können die Fernuniversität Hagen mit ihrem virtuellen Campus, die Open University in England oder ihr kanadisches Pendant, die AthabascaUniversität gezählt werden.

Hier wird Bezug auf die Dual-Mode-Universität, also auf eine Präsenzuniversität genommen und der Frage nachgegangen, welche Möglichkeiten des vernetzten multimedialen Lehrens und Lernens derzeit zur Verfügung stehen. Es werden Überlegungen dazu angestellt, was im erziehungswissenschaftlichen Studium getan werden muß, um die Lehrenden und Studierenden an die neuen Lehr- und Lernformen heranzuführen, damit sie den Anschluß an die globale akademische Gemeinschaft nicht verpassen. 


\section{Gegenwärtige Ausgangsposition an Präsenzuniversitäten: Viele Projekte, kei- ne einheitlichen Konzepte}

Die gegenwärtige Ausgangsposition besteht darin, daß es keine einheitlichen Konzepte des virtuellen Lehrens und Lernens an den Dual-Mode-Universitäten gibt. Die neuen Medien werden durch die Initiative einzelner Dozenten in ihre Lehrveranstaltungen eingebunden. In der Bestandsaufnahme zum medienunterstützten Lehren und Lernen an deutschen Universitäten des HIS der Universität Hannover von 1996 (vgl. Lewin/ Heublein/Kindt/Föge 1996) und auch bei weiteren Recherchen hat sich herausgestellt, daß die meisten Anwendungsprojekte zum Online-Studium in den Naturwissenschaften, der Informatik, Mathematik und den Wirtschaftswissenschaften durchgeführt werden. Im Bereich der Erziehungswissenschaften gibt es wesentlich weniger Projekte, die die neuen Lehr- und Lernformen in ihrer Disziplin einsetzen. ${ }^{1}$

Im erziehungswissenschaftlichen Studium werden häufig neue Lernformen nur aufgrund des Engagements einzelner Dozenten eingeführt wie die Bereitstellung von Vorlesungsmaterialien im Netz oder die Einbindung des Internets als Kommunikationsmedium im Seminar. Während an Universitäten wie Stanford seit 1998 der Master in Elektrotechnik über das Internet erworben werden kann, ist das erziehungswissenschaftliche Diplom noch weit davon entfernt.

Ein zweiter Problembereich besteht darin, daß immer noch viele Studierende in den ersten Semestern kaum Erfahrungen mit dem Internet haben. Dies gilt besonders für Frauen. Und gerade im erziehungswissenschaftlichen Studium sind überproportional viele Frauen anzutreffen.

Daß so wenig Initiative bei der Implementierung neuer Lernformen in der erziehungswissenschaftlichen Disziplin vorhanden ist, liegt m.E. darin, daß immer noch eine Technikdistanz der Lehrenden vorhanden ist, die technische Kompetenz und der Überblick Uber neue Realisierungsmöglichkeiten fehlen. Hinzu kommt sicherlich auch der zu geringe Anreiz für ein Engagement der Dozenten in der Lehre, der auch mit der Furcht vor dem sehr hohen individuellen Aufwand für die Entwicklung und Durchführung der medienunterstützten Lehre verbunden ist (vgl. Lewin/Heublein/Kindt/Föge 1996). Dabei sprechen trotz hohem Organisations- und Herstellungsaufwand eine Reihe von Vorteilen für eine ergänzende „Virtualisierung“ des Lehrens und Lernens im erziehungswissenschaftlichen Studium.

1 Dazu gehören z.B. das Virtual College Berlin (http://www.prz.tu-berlin.de/ virtualc/ index.html) oder das Koalah-Projekt - „Kooperatives Arbeiten und Lernen an der Hochschule“ - an der Universität München (http:// infix.emp.paed.uni-muenchen.de/ nic/seminar.html). 


\section{Vorteile für netzbasiertes Lehren und Lernen im erziehungswissenschaftli- chen Studium}

Obwohl sich die meisten Lehrangebote der Präsenzuniversitäten im Internet noch darauf konzentrieren, Wissen zu archivieren, werden von Multimedia-Anwendungen, Datennetzen und virtuellen Welten weitere didaktische Wirkungsmöglichkeiten erwartet. Dazu gehören beispielsweise eine visuelle Veranschaulichung von Zusammenhängen und Prozessen, ein schnelleres Bereitstellen und Aktualisieren von Informationen, die Überwindung zeitlicher, geographischer und sozialer Grenzen, die Aktivität und Selbststeuerung des Lernenden sowie Kommunikation, Kooperation und Betreuung.

Durch die vernetzten Infrastrukturen kann Wissen in verschiedenen Vorlesungen übermittelt, an verschiedene Universitäten übertragen und viele Studierende können gleichzeitig angesprochen werden. Diese haben dabei die Möglichkeit, Ziele, Inhalte, Dauer und Art ihrer Auseinandersetzung selbst zu bestimmen.

\section{Möglichkeiten des Online-Lehrens und -Lernens}

In den meisten Fällen werden die traditionellen Vermittlungsformen der Lehre nicht durch den Einsatz des Internet ersetzt, sondern ergänzt und unterstützt (vgl. Lewin/Heublein/Kindt/Föge 1996). Neue Lehr- und Lernformen werden damit am meisten komplementär verwendet.

Studierende finden immer häufiger den Vorlesungsstoff im Netz oder können auf Datenbanken und Textsammlungen von überall zugreifen, anstatt jedes Mal in die Bibliothek zu gehen. ${ }^{2}$ In den meisten Lehrveranstaltungen wird die Medienunterstützung fest integriert, aber auch begleitend zu anderen Lehrveranstaltungen angeboten. Es existieren in geringerem Maße auch „freie Angebote, unabhängig von einer Lehrveranstaltung“ (vgl. Lewin/Heublein/Kindt/Föge 1996, 11).

Grundsätzlich sind mittlerweile eine Reihe von asynchronen und synchronen Formen des Medieneinsatzes möglich. Zu den asynchronen Formen des Medieneinsatzes gehören:

- im Netz abrufbare Lernmaterialien, (Vorlesungs-)Skripts, Übungen zu Seminaren, multimedial aufbereitete Lernmodule zur Nachbereitung, Wiederholung und Weiterbearbeitung der Inhalte,

- Email für den Kontakt zwischen Lehrenden und Studierenden,

- Newsgroups, Mailinglists zur kontinuierlichen Beteiligung bei netzbasierten Diskussionsplattformen,

2 Sehr hilfreich für das erziehungswissenschaftliche Studium ist beispielsweise das „Internet-gestützte, integrierte Informations- und Kommunikaüonssystem Bildung“, das von Professor Diepold/HU Berlin betreut wird. 
- „Vorlesungskino“, wo die Vorlesung als Videofilm Uber das Netz abrufbar ist,

- Software-Agenten als „persönliche“ Begleiter und Repräsentanten der Studierenden zur Unterstützung bei der Sammlung von Informationen aus dem Netz.

Zu den synchronen Formen des Medieneinsatzes zählen

- Videokonferenzen, netzbasierte audio-visuelle Sprechstunden, um einzelne Vorlesungen oder Übungen parallel an mehreren Orten anzubieten,

- Desktop-Konferenzen zum gleichzeitigen Konferieren mit mehreren Teilnehmern („Application Sharing“),

- Online-Animationen in großen Lehrveranstaltungen zur Verdeutlichung komplexerer Zusammenhänge,

- MUDs/MOOs zur Gestaltung netzbasierter Lernwelten durch die Teilnehmer, in denen Informationen durch Agenten gesammelt und an andere Mitforschende weitergegeben werden können,

- IRC (Internet Relay Chat) zur Diskussion und Stoffbewältigung,

- Avatare, die als Repräsentanten des Lernenden in einer netzbasierten Umgebung eingesetzt werden.

Insgesamt sind starke Tendenzen zu kommunikativem und kollaborativem Lernen festzustellen. Sie werden unterstützt durch

- Joint Viewing (verschiedene Studierende können an ihren jeweiligen

- Bildschirmen die gleichen Seiten sehen)

- Joint Editing (gemeinsames Erstellen von Texten, Grafiken usw.)

- Application Sharing (gemeinsame Bearbeitung verschiedener Software von verschiedenen Standorten). ${ }^{3}$

\section{Ist die Implementierung didaktischer Konzepte notwendig?}

Grundvoraussetzung für ein erfolgreiches Studium auch im vernetzten virtuellen Raum bleibt die sinnvolle Integration in den Lernkontext. Vernetzte Lern- und Wissensräume, so Schlageter von der Fernuniversität Hagen, müssen kontextorientiert, sozial verankert und komplex statt linear sein. Erfolgreich ist eine Verbindung von individuellem Arbeiten, kooperativen und sozialen Lernprozessen. Notwendig ist auf jeden Fall eine innovative theoretische Konzeption für virtuelle Lernumgebungen an der Präsenzuniversität. Sonst bleiben die Anwendungen immer in einem experimentellen Stadium, wo die technischen Möglichkeiten fasziniert oder frustriert ausprobiert werden. Seminarveranstaltungen des erziehungswissenschaftlichen Studiums könnten beispielsweise wie im Koalah-Projekt-Seminar an der Ludwig-

3 Lernformen nach Dörr/Birkel in Dörr/Jüngst 1998, 146 
Maximilians-Universität nicht mehr nur auf Methoden der Wissensvermittlung, sondern auf konstruktivistische Grundannahmen zurückgreifen.

Konstruktivistische und kognitionspsychologische Erkenntnisse sprechen nämlich derzeit für die Unterstützung und Erleichterung der Lernvorgänge durch hypertextbasiertes Internet: Wissen entsteht über Verknüpfungen in „Cognitive Maps“, Lernerfolge werden durch die stärkere Betonung der Aktivität des Lernenden, durch Aufwerfen von Fragen und durch Entdeckungsprozesse gewonnen, um dann Antworten aus den Informationsverknüpfungen zu finden.

\section{Modularisierung des Wissens in neuen Lernlandschaften}

Gerne werden Powerpoint-Präsentationen ins Netz gestellt. Dabei werden allerdings die Möglichkeiten des Internet nicht annähernd ausgeschöpft. Denn „es kommt nicht darauf an, was ein Student weiß, sondern was er kann“. Diese Aussage stammt von Schank (1994). Schanks Methode basiert auf der Entwicklung von internetgerechten Lerntools, die den Lernenden durch Interaktivität und besonders durch Dialog fesseln. Von Bedeutung sind daher auch Forschungsarbeiten, die Werkzeuge und „Campus-Metaphern“, wie sie die FU Hagen darstellt, für gemeinsame Entwicklungsarbeiten einsetzen wollen. Ein interessantes Beispiel dafür ist auch das FLE-Projekt in Finnland. ${ }^{4}$

Sinnvoll sind Lernformen, die den Forderungen nach Flexibilität, Problemlösefähigkeit, Teamfähigkeit, Informationsverarbeitungskompetenz (Wissensmanagement) entgegen kommen. Die Modularisierung des Wissens kann eine geeignete Methode darstellen, um einen qualitativ hohen Standard der Lernangebote zu erreichen. Es gibt das Modell der „Learning Landscape“, einer Lernlandschaft (z.B. von Siemens), in der Wissen nicht nur vermittelt werden soll, sondern den Teilnehmern Rückmeldungen über Lernfortschritte und bei Problemen gibt. Das Wissen wird modularisiert, die verschiedenen Module zu Kursen zusammengefügt. Der Vorteil liegt in der Wiederverwendbarkeit der Module in verschiedenen Kursen und Fächern. Hierfür ist zum einen ein didaktisch gut durchdachtes Design der Lernumgebung erforderlich, zum anderen muß diese dem Lernenden interaktiv „Feedback“ geben können, was durch den Einsatz von multimedialen Formen des Wissensmanagements z.B. durch intelligente Agenten erreicht werden könnte.

4 In dem finnischen Projekt von Teemu Leinonen wird an Szenarien gearbeitet, die eine dreidimensionale virtuelle Lernwelt darstellen. Dafür werden Module zur Unterstützung von Methoden des kollaborativen Problemlösens, der gemeinsamen Wissenskonstruktion und zur Entwicklung von metakognitiven Fähigkeiten entwickelt (http://www.mlab.uiah.fi/fle/development/index.html). Ein anderes Beispiel für eine innovative virtuelle Lernplattform stellt IKARUS dar, die an der Universität Dortmund entwickelt wurde (http://www. ikarus.uni-dortmund.de). 


\section{Konsequenzen für das erziehungswissenschaftliche Studium}

Was muß im erziehungswissenschaftlichen Studium getan werden, um die Lehrenden und Studierenden an die neuen virtuellen Lehr- und Lernformen heranzuführen? Traditionelle Lehr- und Lernformen im erziehungswissenschaftlichen Studium sollen nicht ersetzt, aber durch neue hypermediale Methoden ergänzt werden. ${ }^{5}$ Das neue Design des Lernstoffs erfordert allerdings eine größere Bereitschaft und Offenheit, miteinander zu lernen und nicht in Monaden zu lehren. Ein wichtiges Ziel ist die Vernetzung der erziehungswissenschaftlichen Disziplin, nicht zu vertauschen mit einer Verschmelzung. Elementare Erkenntnisse der Disziplin brauchen z.B. langfristig nicht mehr von jedem Professor neu vermittelt werden. Als weitere Ansatzpunkte stellen sich dar:

- Gewöhnung an die Dezentralisierung von Wissensprozessen. Bereits in Großunternehmen lösen sich tradierte Formen der Organisation auf, neue dezentrale Produktions- und Dienstleistungsstätten werden aufgebaut (vgl. Alsdorf/Bannwart 1998). Die Präsenzuniversität als öffentliches Unternehmen sollte sich ebenfalls an eine dezentrale Organisation gewöhnen. Damit bleibt sie eine lernende und lebendige Organisation.

- Einsicht der Lehrenden in die Notwendigkeit, virtuelle Lehr- und Lernformen einzusetzen. Dies ist eine wichtige Voraussetzung für einen souveränen Einsatz. Ziel muß die selbstverständliche Integration der Computertechnologien in das erziehungswissenschaftliche Studium sein. Dazu gehört m. E. auch eine stärkere Verankerung der Medienpädagogik in die Prüfungs- und Studienordnung. ${ }^{6}$

- Innovationsbereitschaft ${ }^{7}$. Eines der großen Probleme im erziehungswissenschaftlichen Studium ist die Innovationsbereitschaft, besonders bei den Lehrenden. Die Studierenden allerdings wachsen mit Informationstechnologien auf, besuchen virtuelle Seminare, auch wenn kaum Kenntnisse vorhanden sind. Zur Innovationsbereitschaft gehört Flexibilität, z.B. in der Entwicklung von modularisierten Lerninhalten, um damit auf

5 Wenn multimediale und interaktive Methoden traditionelle Lehrformen ergänzen oder sogar ersetzen, hat dies laut der Gesellschaft für Informatik e.V. eine Veränderung aller Ebenen der Institution Universität zur Folge: Es betrifft das Selbstverständnis der Lehrenden, Formen des Kontakts und der Rückmeldung zwischen den Lehrenden und Studierenden, der Studierenden untereinander, Vorbereitung und Durchführung von Lehre, Steuerung der Lernprozesse, Betreuung der Studierenden, Beziehung zwischen Lehre und Prüfung, weitere organisatorische Aspekte von Lehren und Lernen im Hochschulkontext (vgl. Gesellschaft für Informatik e.V.: Nutzung neuer Medien für die Ausbildung an den Hochschulen. Positionspapier des Fachbereichs Ausbildung und Beruf vom 24.09.96).

6 In der HIS-Bestandsaufnahme wird dies allerdings nur von ca. 40\% für erforderlich gehalten.

7 Vgl. Innovationsperspektiven für multimedial orientierte Erwachsenenbildung aus pädagogischer Sicht von Hagedorn (1998, Bd. 1, 21). 
Modernisierungstendenzen aktuell reagieren zu können. Zu vermeiden sind festgefügte Anteile von Studienschwerpunkten/Studienrichtungen. Kriterien für Innovation sind Grenzüberschreitungen, Vernetzung und praktische Verschränkung vielfältiger Arbeits-, Lern- und Kommunikationsformen. Multidisziplinäre Kooperation wird für innovative Wissensorganisation immer notwendiger, um fundiertes Wissen zu erhalten. Ansonsten läuft die erziehungswissenschaftliche Disziplin Gefahr, vom stärker werdenden globalen Wettbewerb überholt zu werden oder sich, wie Freimuth es formuliert, ,angesichts der vielen innovativ nutzbaren Möglichkeiten zu verzetteln“ (Freimuth/Haritz 1997, 10).

- Akzeptanz einer veränderten Rolle des Lehrenden und des Studierenden. Mit der Zunahme des Online-Lernens kann auf weltweites erziehungswissenschaftliches Wissen zurückgegriffen werden. Der lehrende Erziehungswissenschaftler muß sich von der Vorstellung verabschieden, daß er allein der Fachvermittler ist. Die Grenzen zwischen dem Dozenten als Vermittler von Wissen und dem Studierenden als Rezipienten verwischt zusehends. Auch der Studierende wird Teil des Entstehungsprozesses von Wissen. Es bestehen Tendenzen zu einer studentenorientierten Bildung, weil die Technologien dem Studierenden eine größere persönliche Kontrolle geben, indem sie auf verschiedenen Wegen Zugang zu Informationen haben und sich die Kombinationen wählen können, die zu ihnen passen. Trotzdem bleibt die Betreuung der Studierenden immer noch unerläßlich.

- Aneignung von Kompetenzen durch Aus- und Weiterbildung mit neuen Medien. Als besonders problematisch erweisen sich im erziehungswissenschaftlichen Studium sowohl bei den Lehrenden wie bei den Studierenden der Mangel an einem kompetenten Umgang mit den virtuellen Lehr-/Lernformen in der Planungs-, Umsetzungsund Nutzungsphase. Den Studierenden wird zwar der Zugang zum Internet immer mehr erleichtert. Sie erhalten beispielsweise einen kostenlosen Intemetanschluß über ihre Universität und können die Computerpools benutzen. Aber der Besitz eines Email-Accounts oder die Bereitschaft zur Teilnahme am virtuellen Seminar bedeutet noch kein tatsächliches Einlassen auf die neuen Kommunikations- und Lernformen (vgl. Heddergott 1998). Aufgrund der mangelhaften Kenntnisse im Umgang mit dem Internet sind daher regelmäßige Einführungskurse erforderlich. Denn auch von Pädagogik-Studenten wird zukünftig eine selbständige Navigation durch die virtuelle Wissenswelt und die eigenständige Entwicklung neuer Fähigkeiten erwartet. $\mathrm{Zu}$ den Anforderungen an das erziehungswissenschaftliche Studium gehört daher eine grundlegende Pflichtveranstaltung, in der z.B. Strategien und Verfahren des wissenschaftlichen Arbeitens in Datenbanken, die Navigation, Recherche und wissenschaftliche Kommunikation im Internet vermittelt werden. Dies erweitert ihre Professionalität.

- Globalisierung der Bildung. Die Erziehungswissenschaften müssen sich mehr als bisher an Konzepten und Umsetzungen zum Online-Lernen und 
-Lehren beteiligen. Viel stärker müssen die zunehmenden Bemühungen der kommerziellen und privatwirtschaftlichen Online-Bildung beachtet werden (Gemeint sind z.B. die kommerziellen Bildungsangebote von der New York City Universität für Unternehmen auf der einen Seite und auf der anderen Seite das Vorpreschen kommerzieller Ausbildungsfirmen wie „Knowledge Universe“, vgl. Krempl in heise. de). ${ }^{8}$ Es entsteht ein globaler elektronischer Bildungsmarkt, auf dem die Universitäten miteinander konkurrieren oder kooperieren, Ressourcen teilen. Sie übernehmen die Rolle von Bildungsbrokern, deren Aufgabe in der Bewertung, Auswahl und Erwerb der modular aufgebauten Bildungsbausteine besteht. Und wer hat für die Bewertung von Bildungskonzepten das Knowhow, wenn nicht die erziehungswissenschaftliche Disziplin?

\section{Literatur}

Alsdorf, C./Bannwart, E.: Das virtuelle Unternehmen. Parallelisierung der Arbeit über Virtual Reality im Netz. In: Schwarzer, R. (Hrsg.): Multimedia und Telelernen: Lernen im Cyberspace. Frankfurt New York 1998, 73-86

Dörr, G./BirkeI P.: Multimediales Lernen in der Weiterbildung. In: Dörr, G./Jüngst, K.L. (Hrsg.): Lernen mit Medien. Weinheim München 1998, 141-158

Freimuth, J./Haritz, J.: Personalentwicklung auf dem Wege zum Wissensmanagement? In: Freimuth, J./Haritz, J./Kiefer, B.U. (Hrsg.): Auf dem Weg zum Wissensmanagement. Göttingen 1997.9-24

Heddergott, K.: Lernen im virtuellen Raum. Grundlagen des Tele-Leaming. In: Nispel, A./Stang, R./ Hagedorn, F. (Hrsg.): Pädagogische Innovation mit Multimedia 1. Frankfurt 1998, 44-49

Lewiri, K./Heublein, U./Kindt M./Föge, A.: Bestandsaufnahme zur Organisation medienunterstützter Lehre an Hochschulen. Hannover September 1996, A7/96

Krämer, S.: Was haben die Medien, der Computer und die Realität miteinander zu tun? In: dies. (Hrsg.): Medien Computer Realität. Frankfurt 1998, 9-26

Schank, R.: Bücher zerstören die Erziehung. In: c’t 1994, Heft 4, 56-58

8 Es wird davon gesprochen, daß durch die Globalisierung des Bildungsmarkts die Kriege ums Wissen begonnen haben (vgl. Krempl in heise.de). 